\title{
L'HOMME L'Homme
}

Revue française d'anthropologie

157 | janvier-mars 2001

Représentations et temporalités

\section{Graça Índias Cordeiro, Um lugar na cidade.} Quotidiano, Memória e Representação no Bairro da Bica

Lisboa, Publicações Dom Quixote, 1997, 414 p., bibl., cartes, ill., ph.

(« Portugal de Perto » 37)

\section{Colette Callier-Boisvert}

\section{(2) OpenEdition}

Journals

Édition électronique

URL : http://journals.openedition.org/lhomme/5735

DOI : 10.4000//homme.5735

ISSN : 1953-8103

Éditeur

Éditions de l'EHESS

Édition imprimée

Date de publication : 1 janvier 2001

Pagination : 279-281

ISBN : 2-7132-1357-6

ISSN : 0439-4216

Référence électronique

Colette Callier-Boisvert, « Graça Índias Cordeiro, Um lugar na cidade. Quotidiano, Memória e

Representação no Bairro da Bica », L'Homme [En ligne], 157 | janvier-mars 2001, mis en ligne le 23 mai

2007, consulté le 24 septembre 2020. URL : http://journals.openedition.org//homme/5735 ; DOI :

https://doi.org/10.4000/lhomme.5735

Ce document a été généré automatiquement le 24 septembre 2020.

(c) École des hautes études en sciences sociales 


\title{
Graça Índias Cordeiro, Um lugar na cidade. Quotidiano, Memória e Representação no Bairro da Bica
}

\author{
Lisboa, Publicações Dom Quixote, 1997, 414 p., bibl., cartes, ill., ph.
}

(«Portugal de Perto » 37)

\section{Colette Callier-Boisvert}

1 CE «LIEU DANS LA VILLE » est un ancien quartier de Lisbonne dont le nom évocateur, «Bica», désigne le tuyau par lequel s'écoule l'eau des fontaines, qui y sont très nombreuses. L'étude de ce lieu emblématique de la réalité urbaine sert de point de départ à une interrogation sur les processus de construction de l'imaginaire collectif. En effet, la relation interactive ville/quartier est au centre de la thématique de cet ouvrage sur la production des identités locales urbaines.

2 Les différentes étapes de construction de l'objet de recherche sont intégrées dans la problématique, y compris les inévitables tâtonnements nécessaires pour délimiter et réaliser l'emboîtement des divers niveaux d'observation: ville, commune (freguesia), quartier, chaussée en pente, ruelle. L'enquête est d'ailleurs présentée, avec une grande modestie, comme "exploratoire » en raison des limites qu'imposait la disproportion entre les capacités d'un unique chercheur et l'amplitude du champ urbain d'une capitale. Le terrain est toutefois bien défriché et nombre de questions sont traitées avec pertinence.

3 La démarche de Graça Índias Cordeiro à la fois historique et ethnographique. Le dépouillement systématique de textes, de documents juridiques, mais aussi de la presse périodique de la capitale de 1865 à nos jours, lui a permis de retracer l'histoire sociale et culturelle des habitants. L'auteur a par ailleurs constitué une base de données sur la population et l'habitat du quartier concerné à partir de statistiques démographiques et d'archives urbaines portant sur cette période. Enfin, l'observation ethnographique du quotidien de la rue lui a apporté une connaissance directe de la réalité vivante.

4 La réflexion sur la notion de bairro popular est un thème central de l'ouvrage. Le «quartier populaire » est une construction idéologique qui a permis de faire revivre 
certains quartiers anciens proches du centre et du Tage, parmi les plus misérables de la capitale, en les promouvant comme porteurs de l'authenticité de l'âme lisboète. L'angle d'attaque de cet imaginaire collectif est l'analyse des fêtes des « saints populaires » du mois de juin, à savoir la Saint-Antoine, la Saint- Jean et la Saint-Pierre, qui sont l'occasion des plus grandes manifestations populaires de Lisbonne, amplement diffusées par la presse et la télévision. Ces événements s'élaborent, au sein du quartier, entre les réseaux d'interaction sociale, les responsables des associations de quartier et la municipalité. Au cours de ces fêtes, les marchas populares (cortèges) des différents quartiers symbolisent l'unité culturelle et sociale des communautés, mettant en scène leur cohésion et leur cohérence. Chaque quartier défile en exprimant sa singularité et son identification à Lisbonne à travers diverses figures de sa population: varinas (marchandes de poissons), fadistas (chanteurs de fado), marins, pêcheurs, porteurs d'eau et autres petits métiers de la rue. Les marchas rivalisent les unes avec les autres lors des concours organisés par la municipalité et contribuent à cimenter l'esprit de quartier.

5 Entité sociale et spatiale toujours désignée par un nom, mais sans reconnaissance administrative propre (distincte en cela de la commune, freguesia, division administrative de la ville), le bairro popular connote essentiellement le tourisme, la culture populaire et les racines de l'identité urbaine. Alfama, autre quartier de Lisbonne étudié dans les années 80 par Firmino da Costa, en est l'exemple-type ${ }^{1}$. La Bica est un cas à part. Situé dans un vallon encaissé entre deux collines, perpendiculaire au fleuve, il ne constitue qu'une partie de la freguesia de São Paulo, et la moins connue parce que dissimulée à la vue par une rangée d'immeubles élevés et la voie d'un funiculaire reliant les abords animés de la rive du Tage et la colline de Santa Catarina, tout aussi active. Enclave proche du centre et du fleuve, zones qui fournissent des emplois plus ou moins stables, la Bica est isolé par sa topographie labyrinthique faite de passages étroits, de volées d'escaliers impressionnantes, de ruelles et de patios à forte solidarité vicinale, fermés sur l'extérieur. Ce quartier est surtout un non-lieu dont la visibilité n'apparaît qu'à l'époque des fêtes des saints populaires, où il se donne à voir à la fois dans les défilés organisés en grande pompe par la municipalité sur les artères centrales de Lisbonne, et dans l'arraial (sorte de kermesse) installé le long de ses venelles et de ses chaussées en escalier, abondamment décorées pour l'occasion.

6 Malgré ses dimensions réduites et son manque de visibilité, la Bica a réussi, dans les années 50, à se hisser au premier rang des quartiers anciens de la ville grâce à la créativité et à l'énergie de ses habitants, en proposant une image de la Lisbonne traditionnelle.

7 C'est cette simultanéité paradoxale d'une popularité recherchée et de la relative dissimulation de la réalité concrète que l'auteur se propose d'analyser. Minutieuse et attentive, son enquête est centrée sur la vie associative et la sociabilité quotidienne de la rue, privilégiant comme espace familier de communication les degrés des chaussées, les pas de porte, les fenêtres, la population s'étant montrée très réservée et sourcilleuse pour tout ce qui concerne la sphère du privé.

8 Nous laisserons de côté l'analyse de la population et de son évolution au cours du siècle (origine géographique, profil socioprofessionnel, réseau familial), la description d'un habitat spécifique au relief accidenté et du genre de vie d'une zone urbaine surpeuplée, pourtant fort intéressante, et retiendrons que la faiblesse des ressources et la précarité des conditions de vie confèrent une certaine homogénéité aux habitants. Les femmes 
jouent un rôle essentiel dans la fixation sociale sur le territoire, les hommes dans la représentation du quartier à l'extérieur. À travers les associations sportives et culturelles qu'ils gèrent, ils font la publicité du quartier à l'extérieur et engrangent des gains matériels et symboliques. Les femmes ont en charge le quotidien, produisent et reproduisent une identité locale, comme l'atteste l'étendue de leurs réseaux de parenté.

9 L'approche ethnographique choisie par l'auteur lui permet de brosser des portraits vivants des habitants et de décrire avec précision les structures et les modes de fonctionnement des associations masculines (colectividades). On a là une forme de sociabilité urbaine qui possède une certaine spécificité au Portugal, jusqu'ici trop peu étudiée, et qu'on se réjouit de voir traiter ici. Ces associations ont un rôle déterminant dans la constitution de l'esprit de quartier (bairrismo) ainsi que dans l'appropriation et la gestion de la mémoire collective locale.

10 L'auteur fait ensuite l'historique des fêtes des saints populaires depuis la Renaissance, époque à laquelle s'intensifie la popularité de saint Antoine $^{2}$. S'agit-il d'une manifestation spontanée, ou est-elle vivement encouragée par les autorités municipales? L'emprise des autorités s'est renforcée au fil des siècles. Si les marchas de juin se rapprochent par bien des aspects des défilés carnavalesques, elles ont fini par s'imposer au dépens de ces derniers. Rite de passage entre deux temps, celui du travail et celui du repos, elles marquent l'aspect saisonnier de la vie collective urbaine.

L'étude des pratiques de sociabilité festive et de loisir entre gens de même niveau social fait apparaître les processus de sédimentation de l'histoire locale grâce au partage des mémoires familiales. Les unités sociales fortement territorialisées que sont les associations, les réseaux de voisinage et de parenté construisent et s'approprient une entité - le bairro popular - qui n'est qu'une représentation élaborée par elles-mêmes. Cette image, qui s'intègre dans l'imaginaire collectif de la ville, contribue à la création, annuellement ritualisée dans un spectacle, d'une relative unité culturelle du milieu urbain. C'est ainsi que la construction sociale de Lisbonne, qui incorpore dans un tout des quartiers si divers, fait de la capitale un microcosme de la société nationale.

L'auteur propose une dernière piste de réflexion s'agissant des rapports de forces entre municipalité et quartiers, qu'il serait utile de creuser. Ces derniers tentent de manipuler le pouvoir central à leur avantage à l'occasion du dialogue qui s'instaure, lors de la préparation et pendant la célébration des fêtes de juin, entre deux mondes fortement séparés dans la vie de tous les jours. Pour ces couches sociales dominées, il s'agit d'inventer des stratégies de séduction et de reconnaissance pour obtenir des pouvoirs publics faveurs et protection, lesquelles aident à supporter le quotidien.

13 Pionnier au Portugal, cet ouvrage d'anthropologie urbaine défriche le terrain avec beaucoup de finesse et d'intelligence, et soulève de nombreuses questions. Une analyse comparative avec Porto et ses ilhas (îlots urbains) ${ }^{3}$, les fêtes commémoratives de la Saint-Jean et celles de chaque freguesia de la deuxième ville du pays, serait particulièrement fructueuse et permettrait de confirmer ou au contraire de relativiser certaines hypothèses avancées ici. Ajoutons qu'une annexe expose la méthode suivie pour construire une base de données informatiques sur la population et l'habitat. On trouvera également de nombreuses cartes ainsi que des documents sur les associations et les fêtes lisboètes. Enfin, le livre est illustré de photographies anciennes et récentes de Lisbonne et de la Bica. 


\section{NOTES}

1. Citons, entre autres travaux d'António Firmino da Costa, « Entre o cais e o castelo : identidade cultural num tecido inegualitário ", Revista Crítica de Ciências Sociais, 1984, $14: 77-108$, et « Ambivalências. Formas sociais e culturais no bairro de Alfama », Povos e Culturas, 1988, $3: 169-185$.

2. ... dit « de Padoue ». Religieux franciscain né en 1195 dans les environs de Lisbonne, saint Antoine est considéré comme un des saints protecteurs de la ville.

3. Voir, à titre d'exemple, Gaspar Martins Pereira, « Housing, Household and the Family : the "ilhas" of Porto at the end of the Nineteenth Century ", Journal of Family History, 1994, 19 (3) : 213-236, et Paulo Castro Seixas, «Identidades de uma cidade : as ilhas e o Porto ", in Vítor Oliveira Jorge \& Raúl Iturra, s. dir., Recuperar o espanto : o olhar da antropologia, Porto, Edições Afrontamento, 1997 : 103-115.

\section{AUTEUR}

\section{COLETTE CALLIER-BOISVERT}

EHESS, Centre d'études portugaises, Paris. 